\title{
Versatile and selective fluorination of the surface of polymeric materials after stereolithography 3D printing
}

\author{
Megan A. Catterton, Alyssa N. Montalbine, and Rebecca R. Pompano* \\ Department of Chemistry \\ University of Virginia \\ PO BOX 400319 \\ Charlottesville, VA 22904
}

Tel: 434-982-1825

*Corresponding author: rpompano@virginia.edu 


\section{$\underline{\text { Abstract }}$}

With the microfluidics community embracing 3D resin printing as a rapid fabrication method, controlling surface chemistry has emerged as a new challenge. Fluorination of 3D printed surfaces is highly desirable in many applications due to chemical inertness, low friction coefficients, anti-fouling properties and the potential for selective hydrophobic patterning. Despite sporadic reports, silanization methods have not been optimized for covalent bonding with polymeric resins. As a case study, we tested the silanization of a commercially available (meth)acrylate-based resin (BV-007A) with a fluoroalkyl trichlorosilane. Interestingly, plasma oxidation was unnecessary for silanization of this resin, and indeed was ineffective. Solventbased deposition in a fluorinated oil (FC-40) generated significantly higher contact angles than deposition in ethanol or gas-phase deposition, yielding hydrophobic surfaces with contact angle $>110^{\circ}$ under optimized conditions. Attenuated Total Reflectance-Fourier Transform Infrared (ATR-FTIR) spectroscopy indicated that the increase in contact angle correlated with consumption of a carbonyl moiety, suggesting covalent bonding of the silane without plasma oxidation. Consistent with a covalent bond, the silanization was resistant to mechanical damage and hydrolysis in methanol, and was stable over long-term storage. When tested on a suite of photocrosslinkable resins, this silanization protocol generated highly hydrophobic surfaces (contact angle $>110^{\circ}$ ) on three resins and moderate hydrophobicity $\left(90-100^{\circ}\right)$ on the remainder. Selective patterning of hydrophobic regions in an open 3D-printed microchannel was possible in combination with simple masking techniques. Thus, this facile fluorination strategy is expected to be applicable for resin-printed materials in a variety of contexts including micropatterning and multiphase microfluidics.

Keywords: Two-phase microfluidics, Droplet microfluidics, low surface energy, Digital Light processing (DLP), stereolithography printing (SLA) 


\section{$\underline{\text { Introduction }}$}

As the microfluidics community has increasingly adopted resin 3D printing for device fabrication, including stereolithography (SLA) and digital light processing (DLP) printing, ${ }^{1,2}$ methods to control the surface chemistry of 3D printed devices are emerging as a critical challenge. ${ }^{3}$ In resin printing, UV/visible light is used to cross-link a photocurable, polymeric resin in a layer-by-layer fashion to produce a $3 \mathrm{D}$ structure. ${ }^{1,2}$ While methods for surface functionalization are well established for traditional materials such as glass and polydimethylsiloxane (PDMS), those methods do not necessarily translate directly to the polymeric materials used for 3D printing. A particular challenge is to generate a fluorinated surface on a 3D printed chip. Fluorinated surfaces offer many advantages for microfluidic device design, such as controlled surface wettability for passive fluidic control, chemical inertness, resistance to surface fouling, and low friction coefficient. ${ }^{4-7}$ These properties historically made fluorinated surfaces invaluable for multiphase microfluidic chips. ${ }^{8-13}$ By patterning fluorination amidst a non-fluorinated surface, patterned hydrophobicity has been used to generate droplets, create microarrays, and control microfluidic valving. ${ }^{14-16}$ Therefore, facile methods to selectively fluorinate the surface of polymeric SLA and DLP resins are required, particularly for the commercially available resins used by most laboratories.

Currently, there are few methods available to generate a fluorinated surface on 3D printed material, particularly a patterned surface. One option is to start directly with a fluorinated resin, ${ }^{17}$ but these are rare in practice due to limited commercial options. Additionally, fully fluorinated devices are not readily patterned at the surface due to their chemical inertness. Alternatively, selective surface patterning is possible by using printed pieces modified at the surface with fluorinated coatings. ${ }^{3,18,19}$ Polymeric liquid coatings provide a robust hydrophobic layer up to 
hundreds of micrometers thick, ${ }^{18}$ but may be inappropriate for microscale features that are easily blocked or filled in. A chemical vapor deposition method can be used to generate a thin, highly hydrophobic coating by polymerizing a fluorinated acrylate film on the surface, but has limited use in enclosed channels. ${ }^{20,21}$ Thin coatings can also be achieved by including a polymerization initiator in the resin, to provide covalent anchor points for fluorinated polymer brushes. ${ }^{19}$ However, polymer brushes may exhibit poor mechanical stability during abrasion. ${ }^{3}$

Silanization using fluorinated silanes is a reliable method for molecular-scale surface modification of glass and polydimethylsiloxane (PDMS), ${ }^{22,23}$ but silanization of polymeric materials can be challenging. So far there have been sporadic reports of silanization of resin 3D printed microfluidic devices, e.g. to fluorinate 3D printed molds for $\mathrm{PDMS}^{24}$ and to attach reactive functionalities for bonding of 3D printed pieces. ${ }^{25}$ In some cases, the printed polymer had to be coated with a layer of silica to enable silanization. ${ }^{26,27}$ Extensive surface oxidation is usually required to generate enough silane-reactive functional groups (e.g. hydroxyls) at the polymer surface, but not all polymers can withstand such treatment, as they may degrade after plasma exposure..$^{4,28,29}$ To date, there has been little testing of the conditions required for direct fluoroalkyl silanization of resin printed pieces, nor characterization of the hydrophobicity and stability of the silanized surface.

Here, we aimed to develop a robust and straightforward silanization protocol using (tridecafluoro-1,1,2,2-tetrahydrooctyl) trichlorosilane, a fluoroalkyl silane, and a suite of commercially available SLA and DLP resins to generate a highly fluorinated surface for use in microfluidic devices. While optimizing the reaction conditions to generate the highest possible contact angle, we found, surprisingly, that surface oxidation using air plasma was unnecessary for silanization. To characterize the surface and investigate reactive groups involved in forming a 
covalent bond between the printed resin and the fluoroalkyl silane, we measured the air/water contact angle of the silanized surface and used infrared (IR) spectroscopy. We tested the ability of the method to selectively pattern hydrophobic regions in a 3D printed open microchannel, and further tested the applicability of the optimized method to four additional resins. The method is facile, versatile, and allows for dynamic patterning of a hydrophobic surface on a resin-printed piece.

\section{Experimental Section}

\section{$3 D$ Printing}

Printed parts were designed using Autodesk Inventor 2018. The CAD files were sliced at $50 \mu \mathrm{M}$ intervals using MII Utility Shortcut V 3.27 and printed using a CADworks3D M50-405 printer (MiiCraft, CADworks3D). The commercial resins included were BV-007A (Clear) (MiiCraft, CADworks 3D), Green Master Mold (MiiCraft, CADworks 3D), Dental LT Clear Resin (V2) (FormLabs), and Asiga PlasClear V2 (iMakr). A house-made photoresin consisting of $0.4 \%$ w/v phenylbis(2,4,6-trimethylbenzoyl)phosphineoxide (Irgacure 819) (Therofisher) dissolved in poly(ethylene glycol) diacrylate (PEG-DA) (MW 250) (Sigma Aldrich) was also included in the suite of resins tested. ${ }^{30}$ The printer setting for each resin can be found in Table S1. Printed parts were rinsed with either 95\% ethanol (Koptec), isopropanol (Fisher chemical), or methanol (Fisher chemical) as recommended for by the manufacturer for the resin. Printed pieces were post-cured in an UV-light box, then stored at room temperature on the bench top in polystyrene petri dishes (Fisher) prior to silanization. 


\section{Surface Treatment of 3D Printed Pieces}

Where noted, some printed parts were plasma treated using a BD-20AC laboratory corona treater (Electro-Technic Products, Chicago IL, USA). Printed parts were placed $3 \mathrm{~mm}$ below the plasma source and treated for $5-60 \mathrm{~s}$ immediately prior to surface silanization. For gas-phase deposition, $200 \mu \mathrm{L}$ of neat tridecafluoro-1,1,2,2-tetrahydrooctyl trichlorosilane (Gelest Inc., Morrisville PA, USA) was placed in a vacuum desiccator in a small polypropylene dish, followed immediately by the printed parts, and a vacuum was applied for 2 hours at room temperature. For solvent deposition, the surface of the printed part was submerged in a $10 \% \mathrm{v} / \mathrm{v}$ solution of tridecafluoro-1,1,2,2-tetrahydrooctyl trichlorosilane in solvent (Fluorinert FC-40 (Sigma Aldrich) or 200 proof ethanol (Koptec) for $30 \mathrm{~min}$ at room temperature, unless otherwise specified. After silanization, surfaces were rinsed with 95\% ethanol and DI water and dried with a nitrogen gun.

\section{Contact Angle Measurement}

Surface air/water contact angles were measured using a ramé-hart goniometer (model 200-00, ramé-hart instrument co., Succasunna NJ, USA) and DROPimage Advanced software. Contact angle was measured for 3 separate printed pieces per condition, by pipetting one $5-\mu \mathrm{L}$ droplet of DI water per print onto the silanized surface. $8 \times 8 \times 8 \mathrm{~mm}^{3}$ cubes were used for the printed piece, and oriented so the smooth flat face of the printed cube was tested.

\section{Surface Chemistry Characterization with Infrared Spectroscopy}

The surface chemistry of the printed parts was examined by using an iD7 ATR Nicolet IS5 FT-IR spectrophotometer (Thermo Fischer Scientific). The IR spectrum was measured on the flat smooth face of a 10x10x $2 \mathrm{~mm}^{3}$ printed rectangular prism. The instrument was set to a 
constant gain of 4, and the background was collected prior to each session. Data was collected, visualized, and processed using the OMNIC software (Thermo Fischer Scientific).

\section{Robustness testing}

Printed pieces were silanized according to the optimized method. To test the resistance to mechanical damage, the parts were rubbed against a clean petri dish for $30 \mathrm{~s}$, and the air/water contact angles of the silanized surfaces were measured before and after. To test stability after storage, silanized printed parts were stored in a petri dish at room temperature under ambient light, and the air/water contact angles were repeatedly measured over time. Finally, contact angles were measured before and after soaking the printed parts for 2 hours in methanol.

\section{Selective Patterning of 3D Printed Surfaces}

Rectangular prisms $\left(20 \times 15 \times 3 \mathrm{~mm}^{3}\right)$ were printed using BV-007A resin. Each print contained an embossed cross-shaped open channel with a rectangular cross-section ( $1 \mathrm{~mm}$ deep, $2 \mathrm{~mm}$ wide). Scotch tape (3M) was cut and aligned manually to prevent the fluoroalkyl silane solution from coming into contact with portions of the printed surface inside the channel. Taped pieces were immersed in a solution of $10 \% \mathrm{v} / \mathrm{v}$ (tridecafluoro-1,1,2,2-tetrahydrooctyl) trichlorosilane in FC-40 for $30 \mathrm{~min}$ in a fume hood at room temperature. After treatment, pieces were rinsed with $95 \%$ ethanol and DI water and dried with nitrogen. To test the functionality of the patterned surface, solutions of food coloring in water were pipetted into the arms of the embossed features. 


\section{Data Analysis}

Statistical tests and curve fitting were performed using Graphpad Prism version 9. Halflives and half-times of exponential fits were calculated according to half time $=\ln 2 / k$, where $k$ is the rate constant from the fit.

\section{$\underline{\text { Results and Discussion }}$}

Plasma oxidation was not necessary or effective for silanization of SLA printed pieces

While the precise composition of most commercial resins is proprietary, MSDS information states that many are based on acrylate and/or methacrylate polymers (Figure 1a). Silanization of related polymeric materials such as poly(methyl methacrylate) (PMMA) requires oxidation to generate hydroxyl groups that undergo condensation reactions with the silane reagent. ${ }^{411}$ Similarly, prior reports of silanization of an acrylate-based 3D printed material included activation of the surface with plasma treatment. ${ }^{24,25}$ Therefore, we first tested the efficacy of silanization of 3D printed pieces as a function of the duration of exposure to air plasma. As a case study, we selected a clear (meth)acrylate-based resin formulated specifically for printing microfluidic devices, BV-007A resin from MiiCraft, and sought to silanize it with (tridecafluoro-1,1,2,2-tetrahydrooctyl) trichlorosilane (Figure 1a). Two common methods of silanization were tested: gas-phase deposition ${ }^{24,31-34}$ and liquid-phase deposition. ${ }^{11,31}$ For the latter, we used a 10\% v/v solution of silane in FC-40 fluorinated oil.

Surprisingly, we found that even in the absence of plasma treatment ( 0 s exposure), silanization significantly increased the air/water contact angle for both methods (gas phase, $\mathrm{p}<0.005$; solvent, $\mathrm{p}<0.001)$ compared to the $60^{\circ}$ contact angle of the unslianized printed piece angle (Figure 1b, Figure S1). While gas-phase deposition provided a contact angle near $90^{\circ}$, the 
lower boundary for hydrophobicity, the solution-phase method provided a significantly larger ( $\mathrm{p}$ $<0.0001$ ) contact angle close to $120^{\circ}$, the upper limit for a flat, fluorinated surface. ${ }^{23,35,36}$ Plasma treatment from 5 to $60 \mathrm{~s} \mathrm{did} \mathrm{not} \mathrm{further} \mathrm{increase} \mathrm{the} \mathrm{contact} \mathrm{angle.} \mathrm{Wanting} \mathrm{to} \mathrm{further} \mathrm{test} \mathrm{the}$ impact of plasma cleaning on the surface chemistry, we next used IR spectroscopy to investigate functional groups on the surface of printed BV-007A pieces.

a<smiles>[2H]C(F)(Cl)CC(F)(F)C(F)(F)C(F)(F)C(F)(F)C(F)(F)C(F)(F)F</smiles>

Fluoroalkyl Silane

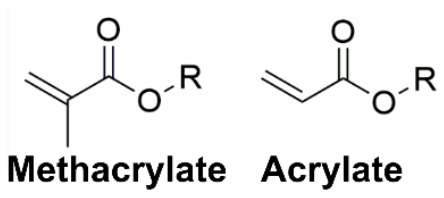

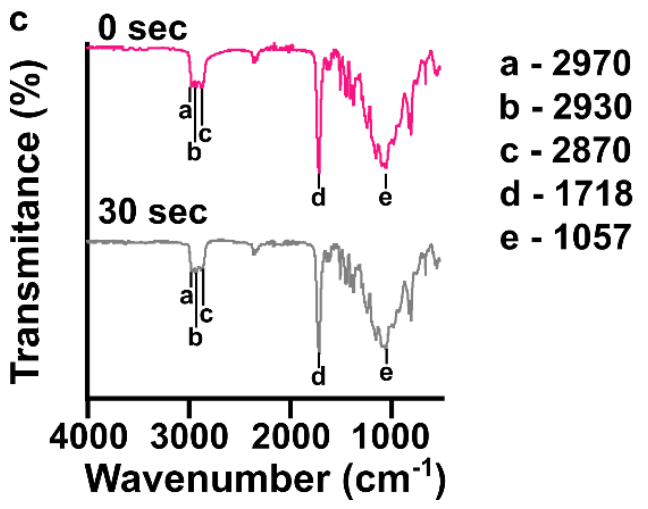

b

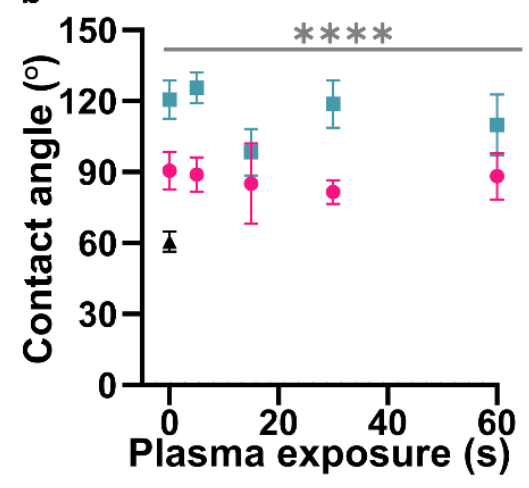

Solvent
deposition

Gas

deposition

$\triangle$ Not Treated

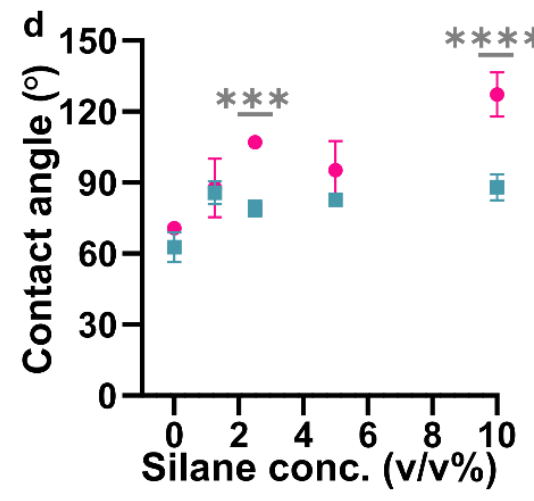

- FC-40

- Ethanol

Figure 1: Effects of plasma treatment and silanization on the chemistry and hydrophobicity of DLP printed pieces. (a) Chemical structures of the fluoroalkyl silane and monomer acylate and methacrylate base used for many resin formulations. (b) Air/water contact angles of BV-007A after silanization by solution-phase (blue squares, FC-40 solvent) or gas-phase (pink dots) deposition after varied times of treatment with air plasma $(n=3$, mean \pm std dev). The black triangle represents printed $B V-007 A$ pieces that received neither plasma treatment nor any silane treatment. Two-way ANOVA for solution vs gas-phase silanization (**** $p<0.0001)$. (c) ATR-FT IR spectrum of the BV-007A surface with no exposure to air plasma (pink) and after 30 s plasma treatment (grey), without silanization. (d) Air/water contact angles of BV-007A surface after solution-phase silanization in FC-40 (pink dot) or ethanol (blue square). Two-way ANOVA with Sidak's multiple comparisons to compare between solvents $(* * * * p<0.0001, * * * p<0.001)$. 
We expected that sufficient exposure of BV-007A pieces to air plasma would oxidize the surface to form alcohol and/or carboxylic acid groups. ${ }^{37}$ To characterize the surface chemistry and investigate the extent of surface activation at short plasma treatment times, we collected surface ATR FI-IR spectra of the printed pieces (Figure 1c). As expected for (meth)acrylatebased BV-007A, the spectra closely resembled that of commercial sheet of PMMA (Figure S2). The peaks at 2970,2930 , and $2870 \mathrm{~cm}^{-1}$ were assigned to alkane $\mathrm{sp}^{3} \mathrm{C}-\mathrm{H}$ stretching. A major $\mathrm{C}=\mathrm{O}$ stretch peak at $1718 \mathrm{~cm}^{-1}$ was attributed to the carbonyl in the backbone of the (meth)acrylate-based polymer as well as other carbonyl-containing components of the resin, e.g. photoinitiators and photoabsorbers. The $\mathrm{C}-\mathrm{O}-\mathrm{C}$ stretching was assigned to the peaks ranging from $1000-1300 \mathrm{~cm}^{-1}$ in the fingerprint region. ${ }^{38}$ Treating BV-007A printed pieces with air plasma for 30 - $60 \mathrm{~s}$ did not alter the IR spectra substantially (Figure 1c and data not shown). In particular, no characteristically broad alcohol band $\left(3550-3200 \mathrm{~cm}^{-1}\right)$ was observed, and there was no change in the alkyl $\mathrm{CH}$ stretches or carbonyl peak. These data were consistent with plasma treatment not affecting the contact angle after silanization (Figure 1b). As a positive control, oxidation from the plasma treatment was verified using both glass and PDMS, whose contact angle decreased after $5 \mathrm{~s}$ of plasma treatment as expected (Table S2). Prior reports of plasma treatment of PMMA used longer treatment times (5 min and greater) to modulate the surface polarity, ${ }^{39,40}$ but we found that treatment of BV-007A pieces with air plasma for longer than 2 min generated cracks in the surface. Since plasma treatment was unnecessary for silanization and in fact was ineffective at oxidizing the BV-007A surface at short times, we proceeded to optimize and characterize the silanization of BV-007A pieces it its absence. 
Solvent deposition was most effective when a fluorocarbon oil was used as a solvent.

Having established that solution-phase deposition was more effective than gas-phase deposition, we further optimized the choice of solvent and concentration of silane. Two solvents were tested: ethanol (200 proof), a common solvent for deposition of trichlorosilanes, ${ }^{31,41}$ and FC-40, a fluorinated oil. ${ }^{11}$ Whereas deposition from ethanol solution was largely ineffective (contact angles $<90^{\circ}$ ) regardless of silane concentration, deposition from FC-40 solution had a concentration-dependent effect, yielding an average contact angle of $\sim 120^{\circ}$ at $10 \% \mathrm{v} / \mathrm{v}$ silane (Figure 1d). Therefore, 10\% v/v of the fluorinated silane in FC-40 was used for all further experiments.

Time dependence of the reaction provides support for covalent bond formation

Next, we tested the time dependence of the silanization reaction. The contact angle increased in a time-dependent manner with a half-time of 3.4 min, reaching a plateau after 15 min (Figure 2a). To complement the contact angle data and assess the extent of bond formation between the fluoroalkyl silane and BV-007A, ATR-FT IR spectra were collected from these samples (Figure 2b). The spectra changed noticeably over this time period. In particular, the carbonyl stretch at $1716 \mathrm{~cm}^{-1}$ decreased in intensity over time (Figure $2 \mathrm{~b}-\mathrm{c}$ ), and the peak area was well fit by exponential decay equation with a half-life of $3.5 \mathrm{~min}$ (Figure $2 \mathrm{~d}$ ). This observation suggested a molecular reaction between the resin and the fluoroalkyl silane that consumes a carbonyl, though the data do not distinguish between the methacrylate carbonyl and any carbonyls that may be present in the resin's photoinitiators or photoabsorbers. An immediate increase in fingerprint region intensity was consistent with the addition of fluoroalkyl silane to the surface of the print (Figure $2 \mathrm{~b}, \mathrm{c}$ and e). New peaks included those at $1023 \mathrm{~cm}^{-1}$, assigned to 

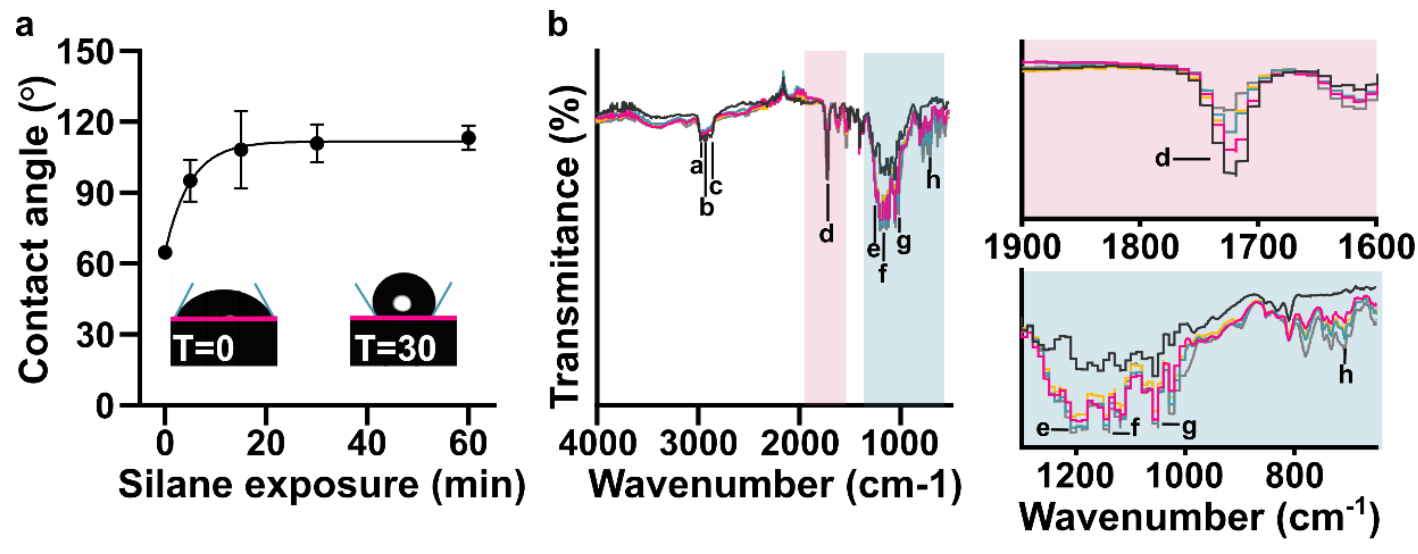

a - 2971

b - 2932

c - 2872

d -1720

e - 1238

f -1143

g -1023

h - 707

- Control

$-5 \mathrm{~min}$

$-15 \mathrm{~min}$

$30 \mathrm{~min}$

$-60 \mathrm{~min}$
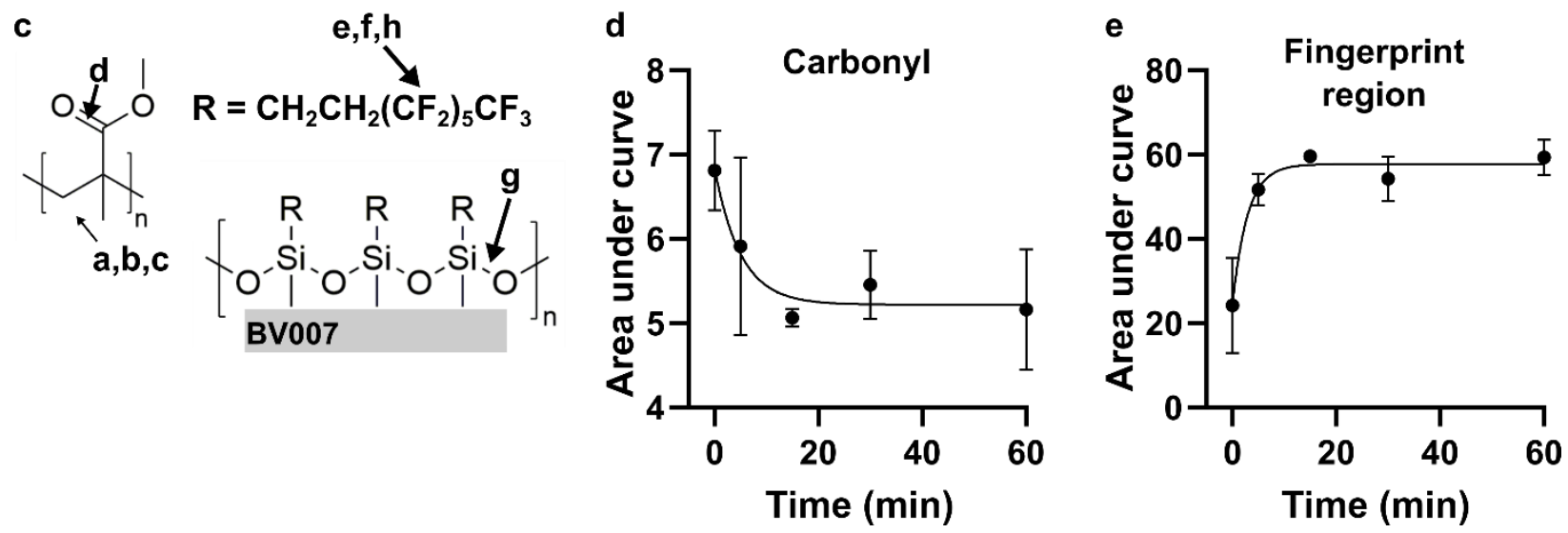

Figure 2: Time dependence of the chemical reaction. (a) Contact angle of the fluorinated surface after various amount of silane treatment $(n=3$, mean \pm std dev). The data were fit to an exponential curve, $y=111-46.9 e^{-0.201 x}, R^{2}=0.844$. Insets show images of droplets on $B V$ 007A surface after 0 and 30 min of silanization. (b) ATR-FT IR spectrum of printed BV-007A pieces after various times of silane treatment. Two regions of interest are highlighted: the carbonyl peak at $1720 \mathrm{~cm}^{-1}$ and the finger print regions $650-1300 \mathrm{~cm}^{-1}$. (c) The chemical structures present in a methyl methacrylate-based resin and from the fluoroalkyl are labeled with the corresponding IR spectra peak. (d) The area under the carbonyl peak decreased in a time-dependent manner $\left(n=3\right.$, mean \pm std dev), fit to an exponential decay $y=55.9 e^{-0.115 x}+$ $43.3, R^{2}=0.936$. (e) The area under the curve of the finger print region increased in a timedependent manner, fit to an exponential curve, $y=57.7-24.2 e^{-0.348 x}, R^{2}=0.855$.

Si-O-R stretching, ${ }^{42} 1232$ and $1142 \mathrm{~cm}^{-1}$, consistent with asymmetric and symmetric C-F stretches, and $707 \mathrm{~cm}^{-1}$, assigned to the C-F wag. ${ }^{43}$ This increase had a half-time of only $2.0 \mathrm{~min}$, shorter than the decay of the carbonyl, suggesting that physical adsorption of the silane may have preceded the covalent reaction (Figure S3). The $-\mathrm{C}-\mathrm{H}$ stretch peaks at 2872, 2932, and $2971 \mathrm{~cm}^{-1}$ 
were still present after silanization (Figure $2 b-c) .{ }^{44}$ From both the contact angle measurements and the IR spectra, we concluded that the silanization reaction likely resulted in a covalent bond, and that 30 min was sufficient for reaction completion and generation of a highly hydrophobic surface.

\section{Robustness and stability of fluorination procedure}

To establish the practical utility of the method, we considered the sensitivity of the procedure to the state of the printed piece and characterized the stability of the hydrophobic surface. First, we considered that the surface chemistry of the printed piece may change over time and potentially alter the reactivity with the trichlorosilane, e.g. due to slow cross-linking of residual monomer under ambient light. ${ }^{45,46}$ To test the efficacy of silanization as a function of light-induced aging, printed parts were treated with either the manufacturer-recommended $20 \mathrm{~s}$ or an extended 360-s UV exposure during the post-curing process. We estimate that continuous 360-s exposure was an equivalent dose of light as being on a bench top under ambient light for 32 days (Table S3). The extended UV cure created discoloration and warped some of the pieces, so only pieces with a flat top surface were used for subsequent silanization. No significant difference was observed in the water contact angles of the control pieces ( $20 \mathrm{~s})$ compared to the pieces with extended UV exposure (360 s), either before or after silanization (Figure 3a). This result was consistent with our informal observations that month-old BV-007A pieces yielded similar contact angles after silanization as recently printed (1-3 days old) pieces. Therefore, the silanization method appears insensitive to the age of the piece, at least in this timescale, which enables robust fabrication procedures. 


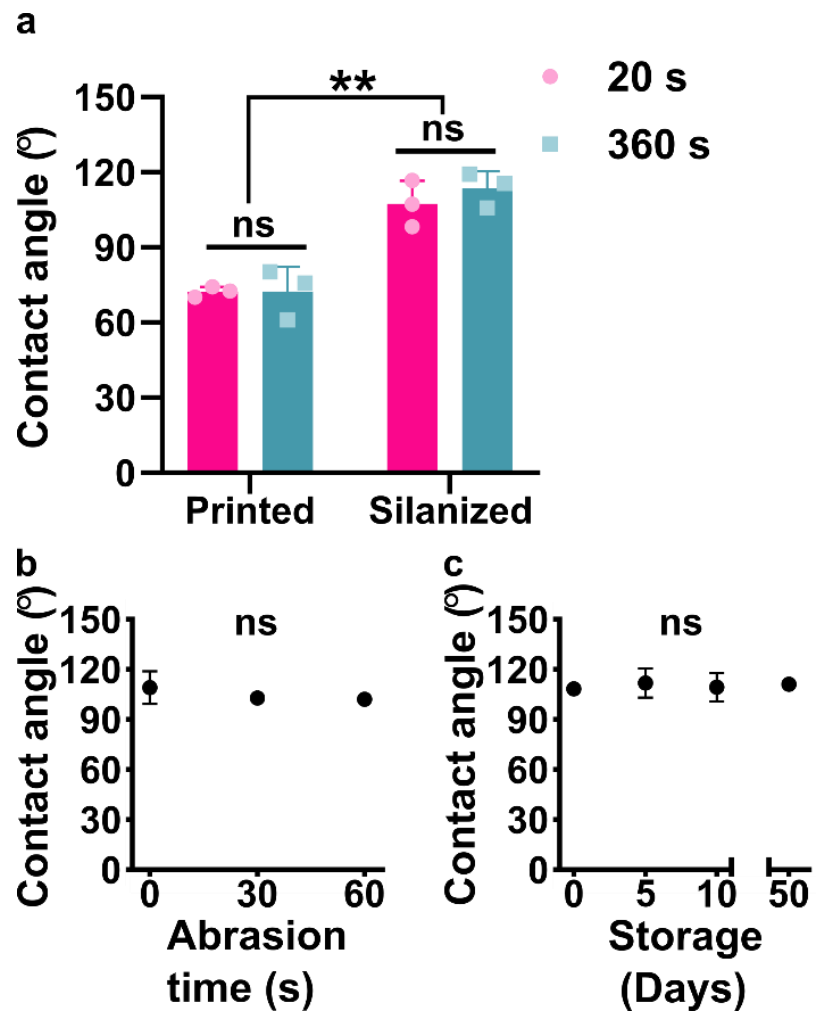

Figure 3: Robustness of the method to the age of the printed piece, abrasion, and storage time after silanization. (a) Contact angle of DLP printed pieces (BV-007A) that were silanized with or without extended $U V$ curing ( $n=3$ printed parts for each condition, mean \pm std dev). Two-way ANOVA with Tukey's multiple comparisons ( $n s, p>0.05$, ** $p<0.005) .(b, c)$ Contact angle of silanized BV007 after deliberate mechanical abrasion $(b)$ or long term storage $(c)(n=3$ printed parts for each condition, mean \pm std dev). One-way ANOVA ( $n s, p>0.05)$.

Next, we assessed the robustness of the silanized surface when subjected to mechanical damage and extended storage, a property that affects the range of potential uses, handling, and storage. Silanized printed pieces were subjected to gentle mechanical damage by manually rubbing the piece against a clean polystyrene surface, mimicking normal wear and tear during use. The water contact angle of the fluorinated pieces of BV-007A was not significant altered by this process (Figure 3b), indicating that the surface is stable under mild abrasion conditions. Similarly, when silanized pieces of BV-007A were stored on the bench, the contact angles remained unchanged for at least 50 days, the longest time point measured (Figure 3c). We did observe that the initial contact angle in these experiments was slightly lower than in previous 
experiments, which we attribute to hydrolysis of the trichlorosilane during storage because replacement of the silane stock improved the hydrophobicity (data not shown). We concluded that the silanized surface was quite stable and the method was robust to the age of the resin though sensitive to the quality of the silane stock, all of which are consistent with the formation of a covalent bond during the silanization reaction.

Patterning of surface hydrophobicity on $3 D$ printed parts

Compared to printing with a fully fluorinated resin, site-specific patterning is an advantage of post-print modifications, offering the potential for passive fluidic control. Therefore, we tested the ability of the silanization protocol to selectively pattern hydrophobic patches on the surface of BV-007A resin, using a pair of intersecting open channels in a simple, recessed cross design. The arms of the cross were protected from the silanization using adhesive tape, while the center square was silanized to generate a pattern of four separate fluid compartments, separated by a surface tension barrier. In the non-silanized control, colored solutions pipetted into the arms of channel mixed readily in the center of the cross (Figure 4a, Not treated), whereas a micropatterned hydrophobic patch in the center of the cross successfully constrained the solutions to the arms (Figure 4a, Pattern). These data demonstrate that because the silanization method requires contact of the liquid silanization solution with the printed surface, it is easily patterned by physical masking strategies to define the silanized area. 
a Not Treated
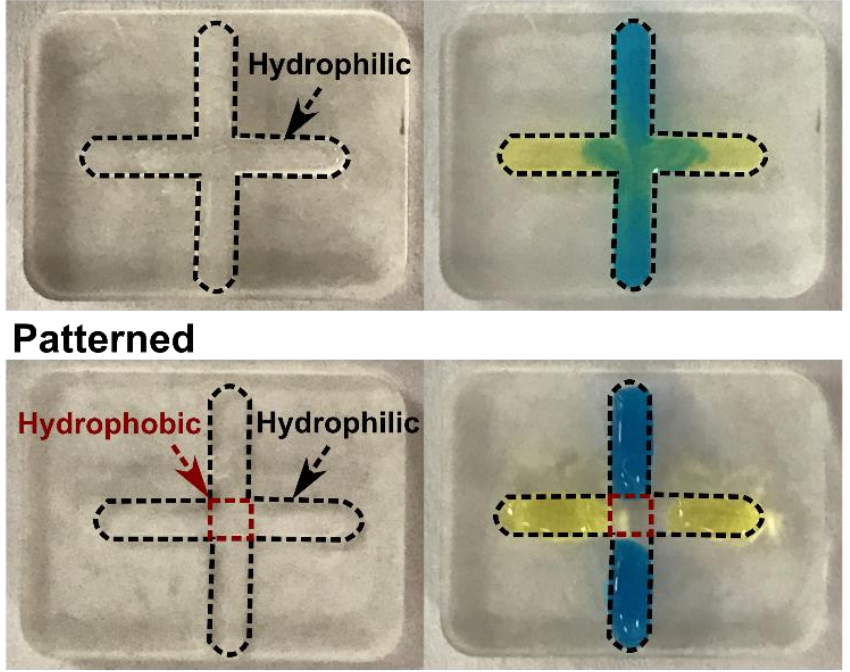

b

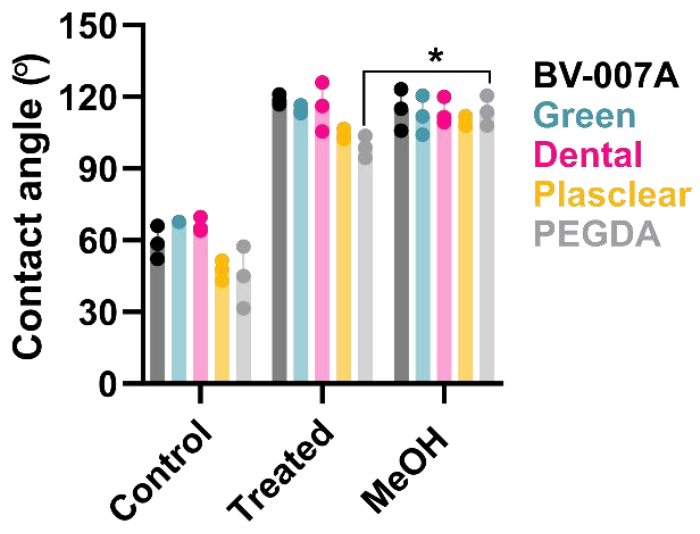

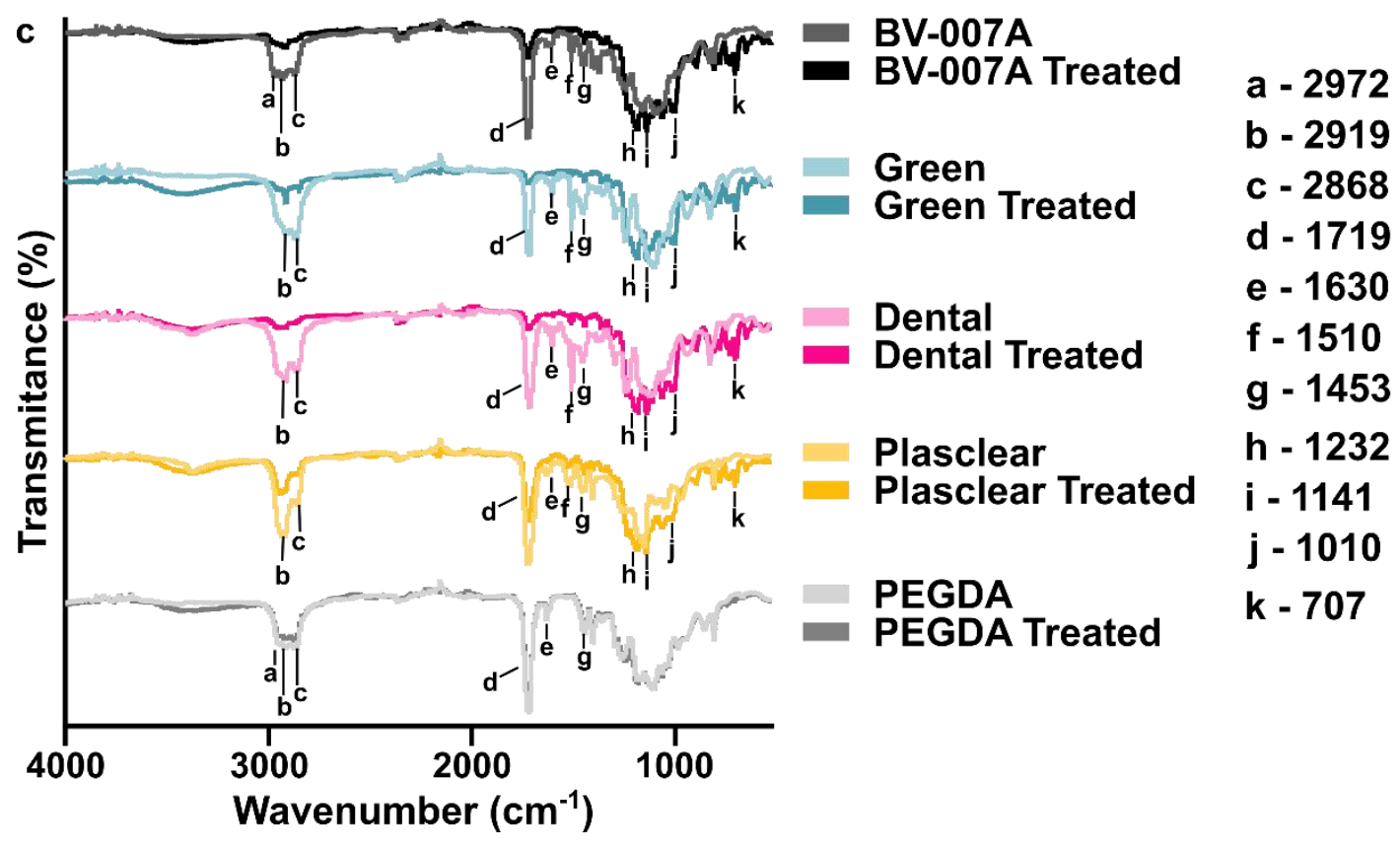

Figure 4: Using the optimized silanization procedure for surface patterning and on other SLA/DLP resins. (a) Photos showing selective surface patterning. Parts printed in BV-007A were patterned so that the center of the cross was hydrophobic. In a non-silanized piece (top), the blue and yellow food dyes mixed in the center; in the piece patterned by local silanization (bottom), the droplets remained distinct from each other. The width of the channels was $2 \mathrm{~mm} .(\mathrm{b})$ Air/water contact angles for resins prior to silanization, after silanization with the optimized procedure, and after soaking in methanol ( $n=3$ printed parts for each condition, mean \pm std dev). Two-way ANOVA with Tukey's multiple comparisons tests ( $n s, p>0.05$, ** p <0.005). (c) ATR FT-IR spectra of pieces printed using the various resins, before and after silanization ( $n=3$ printed parts for each condition). 


\section{Silanization of a suite of SLA resins demonstrates broad applicability}

This silanization protocol would be most useful if applicable across a variety of SLA and DLP resins. Therefore, in addition to BV-007A, we tested three commercially available resins: Dental (FormsLab), Green Master Mold resin (CADworks3D), and Plasclear (iMakr), plus a polyethylene glycol diacrylate (PEG-DA)-based resin developed by the Folch laboratory. ${ }^{30}$ Based on our prior data that the extent of reaction correlated with diminished absorbance from the carbonyl in the IR spectrum, we hypothesized that any resin with an acrylate (BV007A and PEG-DA) or methacrylate (Dental, Green Master Mold, and Plasclear) backbone, or possibly with carbonyl-containing photoinitiators or photoabsorbers, would react with the fluoroalkyl silane. Following the optimized protocol, all printed pieces were submerged in a 10\% (v/v) solution of fluorinated silane in FC-40 oil for $30 \mathrm{~min}$, without plasma treatment. This procedure successfully increased the contact angle for each material compared to its non-treated control (Figure 4b). The Green Master Mold, Dental, and BV007 resins were highly hydrophobic after silanization, with contact angles of $\sim 115-118^{\circ}$. In contrast, the PEG-DA and Plasclear resins had a mildly hydrophobic contact angle, near $100^{\circ}$. This trend was reproduced in two independent experiments. To ensure that any physically adsorbed silane was removed, the silanized pieces were soaked for 2 hours in methanol. ${ }^{47}$ In all cases, the hydrophobic surface persisted, again suggesting that the silane was covalently bound (Figure 4b). Surprisingly, the contact angle increased for both the Plasclear and PEGDA resins, an observation that remains to be explored.

We examined the surface chemistry of the printed pieces by ATR FT-IR to potentially explain the difference in susceptibility to silanization between resins (Figure 4c). The pair of peaks at 1453 and $1510 \mathrm{~cm}^{-1}$ are useful to distinguish PMMA from poly(methyl acrylate) 
(PMA). ${ }^{48}$ The $1453 \mathrm{~cm}^{-1}$ peak, which was present in all samples, is attributable to a methylene vibration -CH2- found in both PMMA and PMA. The peak at $1510 \mathrm{~cm}^{-1}$, attributable to the methyl vibration $\mathrm{C}-\mathrm{CH} 3$, is indicative of PMMA. This peak was present in all four commercial resins tested, suggesting the presence of methacrylates in these materials; as expected, it was not seen in the PEGDA sample. A small peak at $1630 \mathrm{~cm}^{-1}$, assigned to $\mathrm{C}=\mathrm{C}$ bonds from residual (meth)acrylate monomers, ${ }^{49}$ was present in all samples.

Next, changes in the surface IR spectra after silanization were examined. The three resins that exhibited a larger change in contact angle after silanization (BV-007A, Green Master Mold, and Dental) also showed a larger decrease in the intensity of the carbonyl peak at $1719 \mathrm{~cm}^{-1}$ (Figure 4c). Furthermore, the decrease in the carbonyl peak correlated with appearance of peaks consistent with deposition of the fluoroalkyl silane. The peaks at 1232,1142 and $707 \mathrm{~cm}^{-1}$ were again assigned to stretches and wagging of the fluoroalkyl chain, ${ }^{20,43}$ and they increased after silanization for the four commercial resins. Similarly, the peak at $1010 \mathrm{~cm}^{-1}$ that increased after silanization in the commercial resins may be a part of the Si-O-R stretch (usually a strong and broad stretch, 1000-1100 $\left.\mathrm{cm}^{-1}\right) .{ }^{42}$ In contrast, Plasclear and PEGDA, which had smaller changes in contact angle, showed less consumption of the carbonyl, and PEGDA showed no increase in the finger print region. From these data, we concluded that while all five resins showed an increase in contact angle that was resistant to removal by methanol, only a fraction of them formed a covalent bond that consumed a carbonyl. It may be significant that PEGDA, which has no added photoabsorbers, was the least reactive of the materials towards the silane, this possibility was not tested further here. 


\section{$\underline{\text { Conclusions }}$}

We have demonstrated a robust and versatile strategy to control the surface chemistry and hydrophobicity of DLP 3D printed parts by reacting the printed surface with an alkyl-fluorinated silane. This method required only submerging the resin pieces in a silane solution, without any pre-treatment. The reaction between the silane and the resin appeared to consume a carbonyl present in the resin material, and was consistent with covalent bond formation. The fluorinated surface was resistant to mechanical damage, methanol soaking, and 50 days of storage, and the method was compatible with printed parts even after significant light exposure. Selective patterning of a hydrophobic surface was demonstrated in 3D printed open channels by a simple masking method. Furthermore, the method was effective with a suite of (meth)acylate based resins, with higher contact angles correlating with greater consumption of the carbonyl. We anticipate that simple approach to controlling the surface chemistry of resin $3 \mathrm{D}$ printed microfluidic parts, including for selective fluorination of specific regions, will advance the fabrications of complex two-phase devices and enable greater control of the wettability of 3D printed parts.

\section{Acknowledgements}

Research reported in this publication was supported by the National Institute of Allergy and Infectious Diseases under Award Number R01AI131723, and by the National Institute of Biomedical Imaging and Bioengineering under Award Number R03EB028043, both through the National Institutes of Health (NIH). The content is solely the responsibility of the authors and does not necessarily represent the official views of the National Institutes of Health. The authors would like to thank Dr. Tim Allen for access to the goniometer. We also thank Dr. Dean Harman for 
access to the iD7 ATR Nicolet IS5 FT-IR spectrophotometer, Dr. Earl Ashcraft for technical assistance, and Hannah Musgrove for providing the PMMA spectrum.

\section{$\underline{\text { References: }}$}

(1) Bhattacharjee, N.; Urrios, A.; Kang, S.; Folch, A. The Upcoming 3D-Printing Revolution in Microfluidics. Lab Chip 2016, 16 (10), 1720-1742.

https://doi.org/10.1039/C6LC00163G.

(2) Waheed, S.; Cabot, J. M.; Macdonald, N. P.; Lewis, T.; Guijt, R. M.; Paull, B.; Breadmore, M. C. 3D Printed Microfluidic Devices: Enablers and Barriers. Lab Chip 2016, 16 (11), 1993-2013. https://doi.org/10.1039/C6LC00284F.

(3) Jiang, P.; Ji, Z.; Wang, X.; Zhou, F. Surface Functionalization - a New Functional Dimension Added to 3D Printing. J. Mater. Chem. C 2020, 8 (36), 12380-12411. https://doi.org/10.1039/D0TC02850A.

(4) Ward, L. J.; Badyal, J. P. S.; Goodwin, A. J.; Merlin, P. J. Solventless Coupling of Perfluoroalkylchlorosilanes to Atmospheric Plasma Activated Polymer Surfaces. Polymer 2005, 46 (12), 3986-3991. https://doi.org/10.1016/j.polymer.2005.03.009.

(5) Yallup, K.; Iniewski, K. Technologies for Smart Sensors and Sensor Fusion; CRC Press, 2014.

(6) Grover, W. H.; von Muhlen, M. G.; Manalis, S. R. Teflon Films for Chemically-Inert Microfluidic Valves and Pumps. Lab Chip 2008, 8 (6), 913.

https://doi.org/10.1039/b800600h.

(7) Halvey, A. K.; Macdonald, B.; Dhyani, A.; Tuteja, A. Design of Surfaces for Controlling Hard and Soft Fouling. Phil. Trans. R. Soc. A. 2019, 377 (2138), 20180266.

https://doi.org/10.1098/rsta.2018.0266.

(8) Du, W.; Li, L.; Nichols, K. P.; Ismagilov, R. F. SlipChip. Lab Chip 2009, 9 (16), 22862292. https://doi.org/10.1039/B908978K.

(9) Shui, L.; Eijkel, J. C. T.; van den Berg, A. Multiphase Flow in Microfluidic Systems Control and Applications of Droplets and Interfaces. Advances in Colloid and Interface Science 2007, 133 (1), 35-49. https://doi.org/10.1016/j.cis.2007.03.001.

(10) Köster, S.; Angilè, F. E.; Duan, H.; Agresti, J. J.; Wintner, A.; Schmitz, C.; Rowat, A. C.; Merten, C. A.; Pisignano, D.; Griffiths, A. D.; Weitz, D. A. Drop-Based Microfluidic Devices for Encapsulation of Single Cells. Lab Chip 2008, 8 (7), 1110-1115. https://doi.org/10.1039/B802941E. 
(11) Subramanian, B.; Kim, N.; Lee, W.; Spivak, D. A.; Nikitopoulos, D. E.; McCarley, R. L.; Soper, S. A. Surface Modification of Droplet Polymeric Microfluidic Devices for the Stable and Continuous Generation of Aqueous Droplets. Langmuir 2011, 27 (12), 7949_ 7957. https://doi.org/10.1021/la200298n.

(12) Lee, J.; Moon, H.; Fowler, J.; Schoellhammer, T.; Kim, C.-J. Electrowetting and Electrowetting-on-Dielectric for Microscale Liquid Handling. Sensors and Actuators A: Physical 2002, 95 (2), 259-268. https://doi.org/10.1016/S0924-4247(01)00734-8.

(13) J. Jebrail, M.; S. Bartsch, M.; D. Patel, K. Digital Microfluidics: A Versatile Tool for Applications in Chemistry, Biology and Medicine. Lab on a Chip 2012, 12 (14), 24522463. https://doi.org/10.1039/C2LC40318H.

(14) Kobayashi, T.; Shimizu, K.; Kaizuma, Y.; Konishi, S. Novel Combination of Hydrophilic/Hydrophobic Surface for Large Wettability Difference and Its Application to Liquid Manipulation. Lab on a Chip 2011, 11 (4), 639-644. https://doi.org/10.1039/C0LC00394H.

(15) Feng, W.; Ueda, E.; Levkin, P. A. Droplet Microarrays: From Surface Patterning to HighThroughput Applications. Advanced Materials 2018, 30 (20), 1706111. https://doi.org/10.1002/adma.201706111.

(16) Feng, Y.; Zhou, Z.; Ye, X.; Xiong, J. Passive Valves Based on Hydrophobic Microfluidics. Sensors and Actuators A: Physical 2003, 108 (1), 138-143. https://doi.org/10.1016/S0924-4247(03)00363-7.

(17) Kotz, F.; Risch, P.; Helmer, D.; Rapp, B. Highly Fluorinated Methacrylates for Optical 3D Printing of Microfluidic Devices. Micromachines 2018, 9 (3), 115. https://doi.org/10.3390/mi9030115.

(18) Helmer, D.; Keller, N.; Kotz, F.; Stolz, F.; Greiner, C.; Nargang, T. M.; Sachsenheimer, K.; Rapp, B. E. Transparent, Abrasion-Insensitive Superhydrophobic Coatings for RealWorld Applications. Sci Rep 2017, 7 (1), 15078. https://doi.org/10.1038/s41598-01715287-8.

(19) Wang, X.; Cai, X.; Guo, Q.; Zhang, T.; Kobe, B.; Yang, J. I3DP, a Robust 3D Printing Approach Enabling Genetic Post-Printing Surface Modification. Chem. Commun. 2013, 49 (86), 10064-10066. https://doi.org/10.1039/C3CC45817B.

(20) Gupta, M.; Gleason, K. K. Initiated Chemical Vapor Deposition of Poly $(1 \mathrm{H}, 1 \mathrm{H}, 2 \mathrm{H}, 2 \mathrm{H}-$ Perfluorodecyl Acrylate) Thin Films. Langmuir 2006, 22 (24), 10047-10052. https://doi.org/10.1021/la061904m.

(21) Thompson, B.; Riche, C. T.; Movsesian, N.; Bhargava, K. C.; Gupta, M.; Malmstadt, N. Engineered Hydrophobicity of Discrete Microfluidic Elements for Double Emulsion Generation. Microfluid Nanofluid 2016, 20 (5), 78. https://doi.org/10.1007/s10404-0161740-3. 
(22) de Givenchy, E. T.; Amigoni, S.; Martin, C.; Andrada, G.; Caillier, L.; Géribaldi, S.; Guittard, F. Fabrication of Superhydrophobic PDMS Surfaces by Combining Acidic Treatment and Perfluorinated Monolayers. Langmuir 2009, 25 (11), 6448-6453. https://doi.org/10.1021/la900064m.

(23) Nishino, T.; Meguro, M.; Nakamae, K.; Matsushita, M.; Ueda, Y. The Lowest Surface Free Energy Based on -CF3 Alignment. Langmuir 1999, 15 (13), 4321-4323. https://doi.org/10.1021/la981727s.

(24) Waheed, S.; Cabot, J. M.; Macdonald, N. P.; Kalsoom, U.; Farajikhah, S.; Innis, P. C.; Nesterenko, P. N.; Lewis, T. W.; Breadmore, M. C.; Paull, B. Enhanced Physicochemical Properties of Polydimethylsiloxane Based Microfluidic Devices and Thin Films by Incorporating Synthetic Micro-Diamond. Scientific Reports 2017, 7 (1), 15109. https://doi.org/10.1038/s41598-017-15408-3.

(25) Carrell, C. S.; McCord, C. P.; Wydallis, R. M.; Henry, C. S. Sealing 3D-Printed Parts to Poly(Dimethylsiloxane) for Simple Fabrication of Microfluidic Devices. Analytica Chimica Acta 2020, 1124, 78-84. https://doi.org/10.1016/j.aca.2020.05.014.

(26) Ohtani, K.; Tsuchiya, M.; Sugiyama, H.; Katakura, T.; Hayakawa, M.; Kanai, T. Surface Treatment of Flow Channels in Microfluidic Devices Fabricated by Stereolithography. Journal of Oleo Science 2014, 63 (1), 93-96. https://doi.org/10.5650/jos.ess13132.

(27) Anderson, A.; Ashurst, W. R. Enabling Organosilicon Chemistries on Inert Polymer Surfaces with a Vapor-Deposited Silica Layer. Langmuir 2009, 25 (19), 11541-11548. https://doi.org/10.1021/la9014543.

(28) Antonucci, J. M.; Dickens, S. H.; Fowler, B. O.; Xu, H. H. K.; McDonough, W. G. Chemistry of Silanes: Interfaces in Dental Polymers and Composites. J Res Natl Inst Stand Technol 2005, 110 (5), 541-558. https://doi.org/10.6028/jres.110.081.

(29) Weikart, C. M.; Yasuda, H. K. Modification, Degradation, and Stability of Polymeric Surfaces Treated with Reactive Plasmas. Journal of Polymer Science Part A: Polymer Chemistry 2000, 38 (17), 3028-3042. https://doi.org/10.1002/10990518(20000901)38:17<3028::AID-POLA30>3.0.CO;2-B.

(30) Urrios, A.; Parra-Cabrera, C.; Bhattacharjee, N.; Gonzalez-Suarez, A. M.; RigatBrugarolas, L. G.; Nallapatti, U.; Samitier, J.; DeForest, C. A.; Posas, F.; Garcia-Cordero, J. L.; Folch, A. 3D-Printing of Transparent Bio-Microfluidic Devices in PEG-DA. Lab Chip 2016, 16 (12), 2287-2294. https://doi.org/10.1039/c6lc00153j.

(31) Catterton, M. A.; Dunn, A. F.; Pompano, R. R. User-Defined Local Stimulation of Live Tissue through a Movable Microfluidic Port. Lab Chip 2018, 18 (14), 2003-2012. https://doi.org/10.1039/C8LC00204E.

(32) Saini, G.; Sautter, K.; Hild, F. E.; Pauley, J.; Linford, M. R. Two-Silane Chemical Vapor Deposition Treatment of Polymer (Nylon) and Oxide Surfaces That Yields Hydrophobic 
(and Superhydrophobic), Abrasion-Resistant Thin Films. Journal of Vacuum Science \& Technology A 2008, 26 (5), 1224-1234. https://doi.org/10.1116/1.2953699.

(33) Mayer, T. M.; de Boer, M. P.; Shinn, N. D.; Clews, P. J.; Michalske, T. A. Chemical Vapor Deposition of Fluoroalkylsilane Monolayer Films for Adhesion Control in Microelectromechanical Systems. J. Vac. Sci. Technol. B 2000, 18 (5), 2433. https://doi.org/10.1116/1.1288200.

(34) Duffy, D. C.; McDonald, J. C.; Schueller, O. J. A.; Whitesides, G. M. Rapid Prototyping of Microfluidic Systems in Poly(Dimethylsiloxane). Anal. Chem. 1998, 70 (23), 49744984. https://doi.org/10.1021/ac980656z.

(35) Grate, J. W.; Dehoff, K. J.; Warner, M. G.; Pittman, J. W.; Wietsma, T. W.; Zhang, C.; Oostrom, M. Correlation of Oil-Water and Air-Water Contact Angles of Diverse Silanized Surfaces and Relationship to Fluid Interfacial Tensions. Langmuir 2012, 28 (18), 7182-7188. https://doi.org/10.1021/la204322k.

(36) Kim, Y. M.; Arkles, B.; Pan, Y. The Role Of Polarity In The Structure Of Silanes Employed In Surface Modification. In Silanes and Other Coupling Agents, Volume 5; Brill Academic Publishers, 2009; pp 51-64. https://doi.org/10.1163/ej.9789004165915.i348.37.

(37) Ii, E. G.; Barankin, M. D.; Guschl, P. C.; Hicks, R. F. Surface Activation of Poly(Methyl Methacrylate) via Remote Atmospheric Pressure Plasma: Surface Activation of Poly(Methyl Methacrylate) via Remote .... Plasma Processes Polym. 2010, 7 (6), $482-$ 493. https://doi.org/10.1002/ppap.200900113.

(38) Huszank, R.; Szilágyi, E.; Szoboszlai, Z.; Szikszai, Z. Investigation of Chemical Changes in PMMA Induced by 1.6 MeV He+ Irradiation by Ion Beam Analytical Methods (RBSERDA) and Infrared Spectroscopy (ATR-FTIR). Nuclear Instruments and Methods in Physics Research Section B: Beam Interactions with Materials and Atoms 2019, 450, 364368. https://doi.org/10.1016/j.nimb.2018.05.016.

(39) Pawde, S. M.; Deshmukh, K. Surface Characterization of Air Plasma Treated Poly Vinylidene Fluoride and Poly Methyl Methacrylate Films. Polymer Engineering \& Science 2009, 49 (4), 808-818. https://doi.org/10.1002/pen.21319.

(40) Riau, A. K.; Mondal, D.; Yam, G. H. F.; Setiawan, M.; Liedberg, B.; Venkatraman, S. S.; Mehta, J. S. Surface Modification of PMMA to Improve Adhesion to Corneal Substitutes in a Synthetic Core-Skirt Keratoprosthesis. ACS Appl. Mater. Interfaces 2015, 7 (39), 21690-21702. https://doi.org/10.1021/acsami.5b07621.

(41) Tang, L.; Lee, N. Y. A Facile Route for Irreversible Bonding of Plastic-PDMS Hybrid Microdevices at Room Temperature. Lab Chip 2010, 10 (10), 1274-1280. https://doi.org/10.1039/B924753J.

(42) Launer, P.; Arkles, B. Infrared Analysis of Organosilicon Compounds: Spectra-Structure Correlations. Gelest 2013. 
(43) Hoque, E.; DeRose, J. A.; Houriet, R.; Hoffmann, P.; Mathieu, H. J. Stable Perfluorosilane Self-Assembled Monolayers on Copper Oxide Surfaces: Evidence of Siloxy-Copper Bond Formation. Chem. Mater. 2007, 19 (4), 798-804. https://doi.org/10.1021/cm062318h.

(44) Abasi, C. Y.; Wankasi, D.; Dikio, E. D. Adsorption Study of Lead(II) Ions on Poly(Methyl Methacrylate) Waste Material. Asian J. Chem. 2018, 30 (4), 859-867. https://doi.org/10.14233/ajchem.2018.21112.

(45) Tey, J. N.; Soutar, A. M.; Mhaisalkar, S. G.; Yu, H.; Hew, K. M. Mechanical Properties of UV-Curable Polyurethane Acrylate Used in Packaging of MEMS Devices. Thin Solid Films 2006, 504 (1), 384-390. https://doi.org/10.1016/j.tsf.2005.09.104.

(46) Decker, C.; Zahouily, K. Photodegradation and Photooxidation of Thermoset and UVCured Acrylate Polymers. Polymer Degradation and Stability 1999, 64 (2), 293-304. https://doi.org/10.1016/S0141-3910(98)00205-5.

(47) Long, T. M.; Prakash, S.; Shannon, M. A.; Moore, J. S. Water-Vapor Plasma-Based Surface Activation for Trichlorosilane Modification of PMMA. Langmuir 2006, 22 (9), 4104-4109. https://doi.org/10.1021/la052977t.

(48) Kotera, A.; Shima, M.; Akiyama, K.; Kume, M.; Miyakawa, M. Copolymerization Characteristics of Methyl Acrylate and Methyl Methacrylate. II. Analysis of Methyl Acrylate-Methyl Methacrylate Copolymers by Infrared Spectroscopy. BCSJ 1966, 39 (4), 758-761. https://doi.org/10.1246/bcsj.39.758.

(49) Hadavand, B. S.; Hosseini, H. Investigation of Viscoelastic Properties and Thermal Behavior of Photocurable Epoxy Acrylate Nanocomposites. Science and Engineering of Composite Materials 2017, 24 (5), 691-697. https://doi.org/10.1515/secm-2015-0161. 